\title{
Classification of brain processing indicators in financial officers
}

\author{
Phakkharawat Sittiprapaporn ${ }^{1,2}$, Arthur F. Carmazzi ${ }^{3}$ \\ ${ }^{1}$ Assistant Professor, Brain Science and Engineering Innovation Research Group, Mae Fah Luang University, Bangkok, \\ Thailand, ${ }^{2}$ Department of Anti-Aging and Regenerative Science, School of Anti-Aging and Regenerative Medicine, Mae \\ Fah Luang University, Bangkok, Thailand, ${ }^{3}$ Founder, Directive Communication Psychology, Leadership Development \\ and Organizational Change, Bali, Indonesia
}

Background: Arthur F. Carmazzi discovered new way of identifying the human brain's clarity based on the foundations of genetic neurosciences. The specific terminology of "Ambiguity Relief" was used to refer to the human brain's processing that supports evaluating information to relate new context to existing knowledge, simplifying ideas, making decisions, and problem solving into actionable elements. Aim and Objective: This study identified the relationship between brain processing indicator and communication of financial officers in Thailand by using the Colored Brain Communication Inventory. Materials and Methods: Fifty participants were involved in this study. All participants were assessed by using the Colored Brain Communication Inventory developed by Arthur F. Carmazzi. Results: All participants were thirty-one males $(62 \%)$ and nineteen females (38\%). There were eleven persons worked as Manager, considered $25 \%$. Nine participants worked as Director and seven participants worked as Deputy Director and Senior Manager were about 18\%, 14\%, and 14\%, respectively. Five participants worked as Senior Director were in the amount of $10 \%$ of all the participants while two participants worked as Senior Officer and Officer were 4\% and 4\%, respectively. Of the fifty participants, the highest number of 28 participants (56\%) were reported as Green Brain Processing while 12 participants (24\%) were reported as Purple Brain Processing. The less of 7 participants $(14 \%)$ were reported as Red Brain Processing while only 3 participants $(6 \%)$ were reported as Blue Brain Processing, respectively. Conclusion: This study is the first study to explore the effects of brain processing indicator and communication on the leadership and working environment by using Colored Brain Communication Inventory tool in Thailand. By adopting conceptual and hypotheses of Colored Brain model, any awareness by participants might help their team members adopt a cooperation attitude instead of trying to change others beyond their own processes which creating frustration on both sides.

Key words: Brain processing interpretation; Colored brain; Communication; Working environment

\section{INTRODUCTION}

In 2018, Arthur F. Carmazzi discovered new way of identifying the human brain's clarity based on the foundations of genetic neurosciences. ${ }^{1}$ In Carmazzi's study, he proposed the specific terminology of "Ambiguity Relief" to refer to the human brain's processing that supports evaluating information to relate new context to existing knowledge, simplifying ideas, making decisions, and problem solving into actionable elements. ${ }^{1,2}$ The actionable element was claimed to be as an essential element of human communication whereby directly related to action taking and to the attainment of clarity. ${ }^{1}$ As mentioned by Carmazzi, all decisions, innovations, idea generation, memory retention, interactions, and interpretations are filtered through the brain's ambiguity relief process. ${ }^{2,3}$ In addition, the implications of identifying and understanding the brains ambiguity relief process will support improved

\section{Address for Correspondence:}

Dr. Phakkharawat Sittiprapaporn, Assistant Professor, Brain Science and Engineering Innovation Research Group, School of Anti-Aging and Regenerative Medicine, Mae Fah Luang University, Bangkok 10110, Thailand. E-mail: wichian.sit@mfu.ac.th;

Arthur F. Carmazzi, Founder of the Directive Communication Psychology, Leadership Development and Organizational Change, Bali, Indonesia. E-mail: arthur@directivecommunication.com 
communication, cooperation, synergy and how to move people passionately to action. ${ }^{2}$ Therefore, the application of Carmazzi's discovery could be applied to management, marketing and improving relationships as well as getting people to take action, respectively. ${ }^{1-3}$

According to Carmazzi, as we obtained information through environment differently, we then process all information and communication style based on how we think and our brain genetic processor. ${ }^{2}$ Our genetic brain processor is able determine our character and communication style. ${ }^{2}$ Historically, Carmazzi studied three major studies including Herrmann's clinical study in 1972, Segal and Horne's observatory study in 1979 and Cloninger's pathological study in 1994, and found that there were some similarities as well as discrepancies in these previous studies in order to get a more factual representation. ${ }^{2-5}$ Carmazzi's studies revealed a practical model of how people were distinguished from others by their own specific way of processing the world around them, processing information, learning and problem-solving, communicating, and relating to others. With this model, sixty different corporates and six cultural environments with over 8,000 people were statistically tested. $^{2}$ He hypothesizes that people are distinguished from others by their own specific way of processing the world around them, processing information, learning, solving problems, communicating and relating to others. ${ }^{2,6}$ He has then identified four detailed primary genetic brain communication processes which were correlated with these previous studies to develop a profiling instrument known as the Colored Brain Communication Inventory or $\mathrm{CBCI}$ to identify the brain processing indicator. ${ }^{6-8}$ This brain processing indicator comprises four different brain colors including Green Brain, Red Brain, Purple Brain, and Blue Brain processing. This brain processing indicators are used to describe the different characteristics of our genetic processing and mental flexibility or communication style. However, Caramazzi stated that the CBCI is NOT a test for neither behavior nor personality, but an inherent way the brain processes and communicates. ${ }^{2,7,9}$

Moreover, Carmazzi emphasized that with different brain colors, we might have a direct impact on our communication with others such as miscommunications and misunderstandings. Based on Carmazzi's studies, misunderstandings often arise from misunderstanding a person's processes towards objectives or goals and prevent getting everyone speaking the same language. These communications may affect our working relationship with other people especially our work performance. ${ }^{2}$ The Colored Brain model was designed to support the reduction of barriers to create easier, faster, and more effective communication across any group or relationship. Thus, by understanding our brain colors and the distinctive communication characteristics, we will be able to develop appropriate personal communication strategies to capitalize on our strengths, as well as leveraging on the strengths of others to overcome our shortcomings. ${ }^{2,7,9,10}$ According to Carmazzi's study, miscommunication color(s) referred people who make us crazy and need to become more flexible. ${ }^{10}$ In clarification of miscommunication color $(\mathrm{s})$, Carmazzi ${ }^{10}$ explained that if we have a higher concentration in the other color section, then we might have a difficult time in relating to people with that color orientation. Moreover, we might be misinterpreting our action process based on our view instead of leveraging on our strengths. ${ }^{7,10}$ Further insights and direct color relationships could be found at www.carmazzi.net or www.directivecommunication.net.

Generally, many scholars and scientist as well as professional trainers have learned that the Directive Communication ${ }^{\mathrm{TM}}$ Colored Brain technology was developed by Arthur F. Carmazzi based on three separated bodies including (a) Cloninger's study in 1994 on temperament and character involving four years of modern genetic research, (b) Seagal and Horn's study in 1979 involving more than 50,000 people representing over 25 cultures and identifying inherent distinctions in the genetic functioning of people as whole mental - emotional - physical systems, and (c) Herrmann's research on whole brain thinking since 1972, respectively. ${ }^{2-5}$ Arthur F. Carmazzi is recently known as the Principal Founder of the Directive Communication Methodology and ranked as one of the world's top 10 Leadership Professionals by Global Gurus International. ${ }^{2}$

Therefore, the purpose of this study was to explore the relationship between brain processing indicator and communication of financial officers in Thailand. It was the first study to identify the effects of brain processing indicator and communication on the leadership and working performance by using the Colored Brain Communication Inventory in Thailand.

\section{MATERIALS AND METHODS}

\section{Participants}

The study was done with fifty participants, who were working at the management level in the financial institution in Thailand. All participants agreed to be assessed their way of thinking processing using Colored Brain Communication Inventory (CBCI).

\section{Colored Brain Communication Inventory (CBCI)}

Colored Brain Communication Inventory (CBCI) is the tool to identify and categorize those different Brain Communication Processes. ${ }^{7}$ It is a profiling tool and different from other tools as the focus of this tool addresses both "Nature" and "Nurture" in the formation of processing 
characteristics in discovering the Genetic architecture that supports brain processing. This tool also establishes the "Brain Flexibility" (unnatural characteristics that have been developed through environment) that crosses into the realm of environmental emotional factors. ${ }^{2,6-8}$ There are four measured criterion in CBCI including (a) Natural mental processing strength: the natural genetic strengths that determine how the candidate's brain processes information, and how he/she will take action in a given career situation, (b) Learned mental processing strengths: the learned ability to get results in an area unrelated to the candidates natural brain processing, (c) Mental Flexibility: the ability to productively work and communicate with others that operate with different mental processors, and (d) Communication improvement areas: determining what brain processing types are difficult for a candidate to mix with is instrumental in preventing potential problems in management and teamwork, respectively. ${ }^{6-8}$

\section{Statistical analysis}

Analyzing the basic demographic data of participants by using descriptive analysis with qualitative data summarized in term of frequency and percentage. Descriptive analyses were performed for demographics utilizing characteristic measures. Using inferential statistic compared each colored brain and miscommunication color frequency and percentage.

\section{RESULTS AND DISCUSSION}

\section{Demographic information of the participants}

Fifty participants were involved in this study. The demographic data were shown in Table 1. All participants were thirty-one males (62\%) and nineteen females (38\%), respectively. There were eleven persons worked as "Manager" position, considered 225\%. Nine participants worked as "Director" position and seven participants worked as "Deputy Director" and "Senior Manager" positions were about $18 \%$ and $14 \%$ of all participants, respectively. Five participants worked as "Senior Director" were in the amount of $10 \%$ of all the participants while two participants worked as "Senior Officer" and "Officer" positions were $4 \%$ and $4 \%$, respectively.

\section{Colored brain categories and brain processing} indicators

The following were numbers of different brain colors or the communication viewed by all participants. Each color with each total score were participants' foundational communication characteristics. Scores in each color represented the number of participants who had their similar flexibility in the same areas. Of the fifty participants who were assessed by the $\mathrm{CBCI}$, the highest number of 28 participants or $56 \%$ of the participants were reported as "Green Brain Processing" while about 12 participants

\begin{tabular}{lcc}
$\begin{array}{l}\text { Table 1: Demographic information of the } \\
\text { participants }\end{array}$ & N & Percentage \\
\hline Characteristics & & \\
\hline Gender & 31 & 62 \\
Male & 19 & 38 \\
$\quad$ Female & & \\
Position & 5 & 10 \\
Senior Director & 9 & 18 \\
Director & 7 & 14 \\
Deputy Director & 7 & 14 \\
Senior Manager & 11 & 22 \\
Manager & 7 & 14 \\
Assistant Manager & 2 & 4 \\
Senior Officer & 2 & 4 \\
Officer & 50 & 100 \\
Total & & \\
\hline
\end{tabular}

or $24 \%$ of the participants were reported as "Purple Brain Processing". The less of 7 participants or $14 \%$ of the participants were reported as "Red Brain Processing" while only 3 participants or $6 \%$ of the participants were reported as "Blue Brain Processing", respectively (Figure 1).

According to Figure 1, Carmazzi implied all brain processing indicators consisting four different colored brains and their specific characteristics as followed ${ }^{1}$ :

\section{Green Brain processing indicator}

Participants with green brain processing $(56 \%)$ in the present study were characterized by the following characteristics: (a) processing their environment as a summary of the overall situation, and seeing the "big picture" of things they were involved in, (b) thinking as in non-linear random chunks where nothing was connected. As such, they were able to adopt multiple ways of approaching a task, (c) being action sequences by collecting a little information, immediately taking action, and processing information and actions, (d) interacting with others in exchanging ideas to help and to assist in making sense of their direction, (e) being able to work well with others as well as independently, collaborating with others easily to produce creative approaches to deal with an issue and having a big picture outcome in mind when they start a project, (f) being tackle a problem immediately, and (h) usually asking many questions to attempt to understand things objectively, respectively.

\section{Red Brain processing indicator}

Participants with red brain processing (14\%) in the present study were characterized by the following characteristics: (a) having a clear sense of purpose and direction because of their linear cross referencing nature, (b) tending to analyze situations and dealing with issues, one at a time, in order to be very clear and precise in their communication with others, (c) being detailed-oriented by enjoying gathering information, process and shape the information obtained in a structured way, (d) being able to process information 


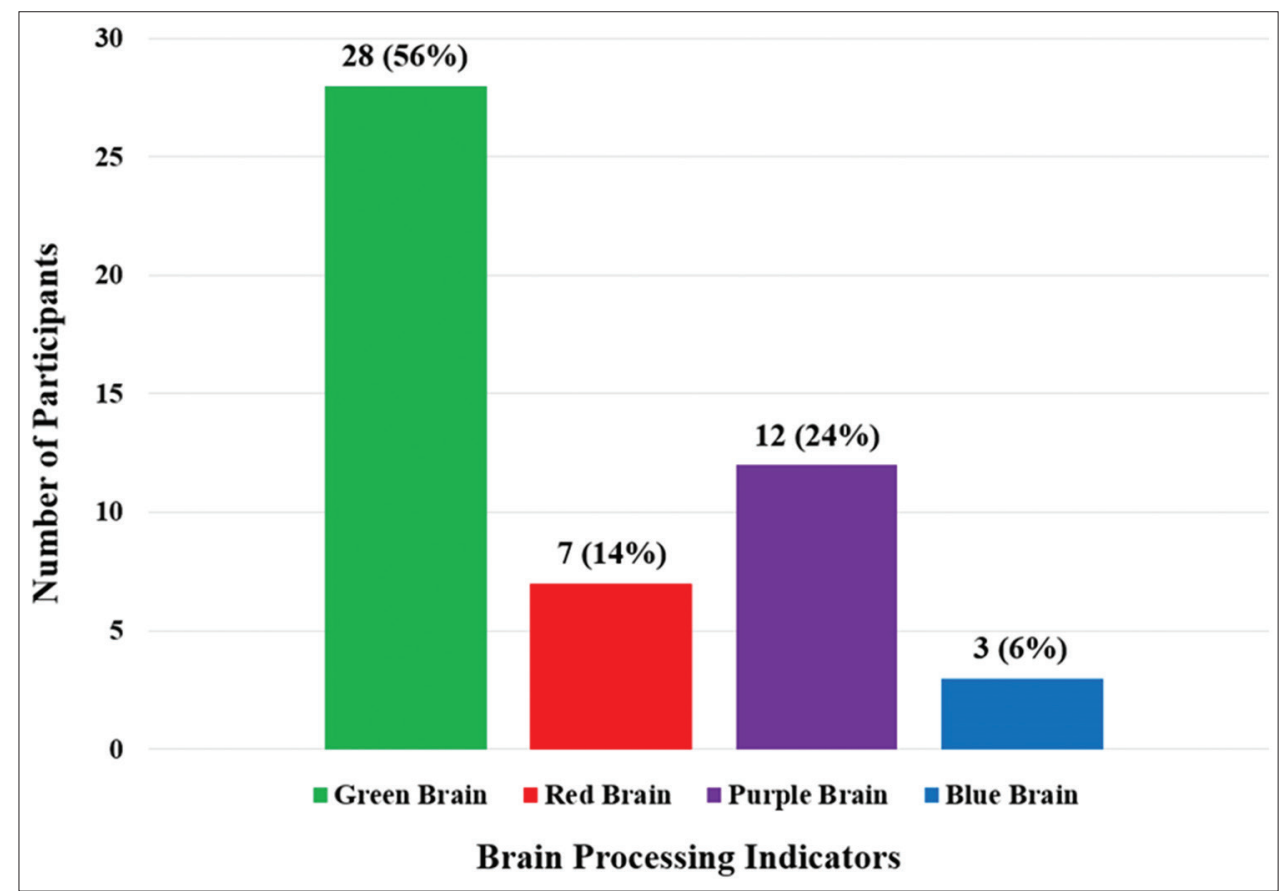

Figure 1: Colored brain categories and brain processing indicators as communication viewed by all involved participants

and facts logically as their objectives, (e) usually not being very expressive and being able to remain calm despite being in a stressful situation, (f) being process information from a long term perspective and often look in the future, (g) being like to deliberate on all the issues and solutions thoroughly before making any decision as they tend to work alone, respectively.

\section{Purple Brain processing indicator}

Participants with purple brain processing (24\%) in the present study were characterized by the following characteristics: (a) being value information a lot and thrive on it, (b) tending to take in a lot of information before making any decision or taking any action, (c) collecting as much information as possible and connecting the information obtained with time, tasks, people, etc. on a framework, (d) needing details information from a different perspective to enable them to make a good decision, (e) being able to accumulate information and encoding it for future use as they often have a sense of individual identity, (f) their way of learning was to absorb a significant amount of information and have ample time to digest the information obtained in order to understand the context clearly and communicate it to others, (g) usually more incline to follow rules due to their orderly nature, respectively.

\section{Blue Brain processing indicator}

Participants with blue brain processing $(6 \%)$ in the present study were characterized by the following characteristics: (a) being more intuitive in nature, (b) being sensitive to their surroundings and the feelings of others as they could be emphatic, (c) being flexible and capable of playing multiple roles, (d) being able to multi-task quite well and being organized, (e) being sensitive to others' feelings and might find it hard to differentiate their own feelings from others, (f) relationship and people were vital in their life preferring to develop a personal connection with the people they work or communicate with, $(\mathrm{g})$ being able to communicate at a deeper and personal level with others because of their expressiveness and intuition, respectively.

\section{Miscommunication color(s) identification}

The number represents higher and lower concentration of each colored brain processing indicator that might have a difficult time in relating to others. Each colored brain processing might be misinterpreting their action processes based on their own views instead of leveraging on their strengths to others. According to Table 2, the highest concentration is 15, implying that the Blue Brain processing participants might have potential miscommunication with Purple Brain processing participants. On the other hand, by showing the lowest concentration of 0 , the Green Brain processing participants do not have any potential miscommunication with Green, Red, and Blue Brain processing participants, respectively.

\section{Blue Brain processing indicator}

Of the three participants who were identified as Blue Brain processing (Figure 1), these three Blue Brain processing participants might have a primary miscommunication with only Purple Brain processing participants (3 persons) (Table 2 and Figure 2). 
According to figure 2, the graph shows a high concentration in only Purple section, implying that these three Blue Brain processing participants might have a difficult time in relating to participant with Purple orientation. In addition, these three Blue Brain processing participants might be misinterpreting their action process based on their own views instead of leveraging on their strengths to Purple Brain processing participants.

\section{Red Brain processing indicator}

Of the seven participants who were identified as Red Brain processing (Figure 1), these seven Red Brain processing participants might have a high primary miscommunication with Green Brain processing participants (3 persons), Purple Brain processing participants (3 persons), Red Brain processing participants ( 2 persons), and Blue Brain processing participants (1 person), respectively (Table 2 and Figure 3).

According to figure 3, the graph shows a higher concentration in both Green and Purple sections, while a

\begin{tabular}{|c|c|c|c|c|}
\hline \multirow{2}{*}{$\begin{array}{l}\text { Colored Brain } \\
\text { Processing } \\
\text { Indicators }\end{array}$} & \multicolumn{4}{|c|}{ Miscommunication Color(s) Identification } \\
\hline & $\begin{array}{l}\text { Green } \\
\text { brain }\end{array}$ & $\begin{array}{l}\text { Red } \\
\text { brain }\end{array}$ & $\begin{array}{l}\text { Purple } \\
\text { brain }\end{array}$ & $\begin{array}{l}\text { Blue } \\
\text { brain }\end{array}$ \\
\hline Green Brain & 0 & 0 & 3 & 0 \\
\hline Red Brain & 3 & 2 & 3 & 1 \\
\hline Purple Brain & 4 & 1 & 5 & 3 \\
\hline Blue Brain & 6 & 5 & 15 & 5 \\
\hline
\end{tabular}

lowest concentration in Blue section, implying that these seven Red Brain processing participants might have a difficult time in relating to participants with both Green and Purple orientation. In addition, these seven Red Brain processing participants might be misinterpreting their action process based on their own views instead of leveraging on their strengths to both Green and Purple Brain processing participants.

\section{Purple Brain processing indicator}

Of the twelve participants who were identified as Purple Brain processing (Figure 1), these twelve Purple Brain processing participants might have a high primary miscommunication with Green Brain processing participants (4 persons), Red Brain processing participant (1 person), Purple Brain processing participants (5 persons), and Blue Brain processing participants ( 3 person), respectively (Table 2 and Figure 4).

According to figure 4, the graph shows a highest concentration in Purple section followed by Green and Blue sections, while a lowest concentration in Red section, implying that these twelve Purple Brain processing participants might have most difficult time in relating to participants with Purple orientation followed by Green, Blue, and Red orientation, respectively. In addition, these twelve Purple Brain processing participants might be misinterpreting their action process based on their own views instead of leveraging on their strengths to Purple, Green, Blue, and Red Brain processing participants, respectively.

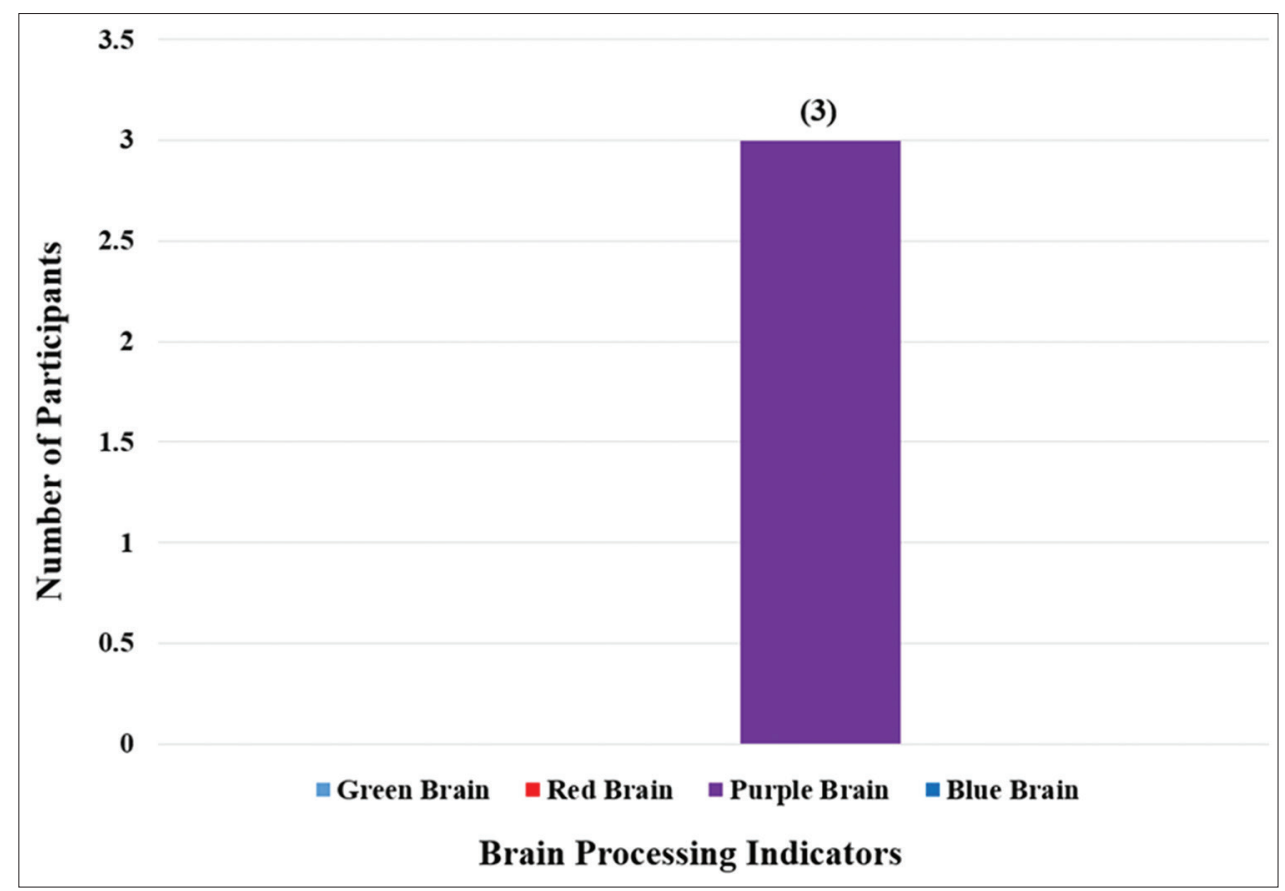

Figure 2: Blue brain processing participants with potentially miscommunication to those participants who were identified as Purple Brain processing 


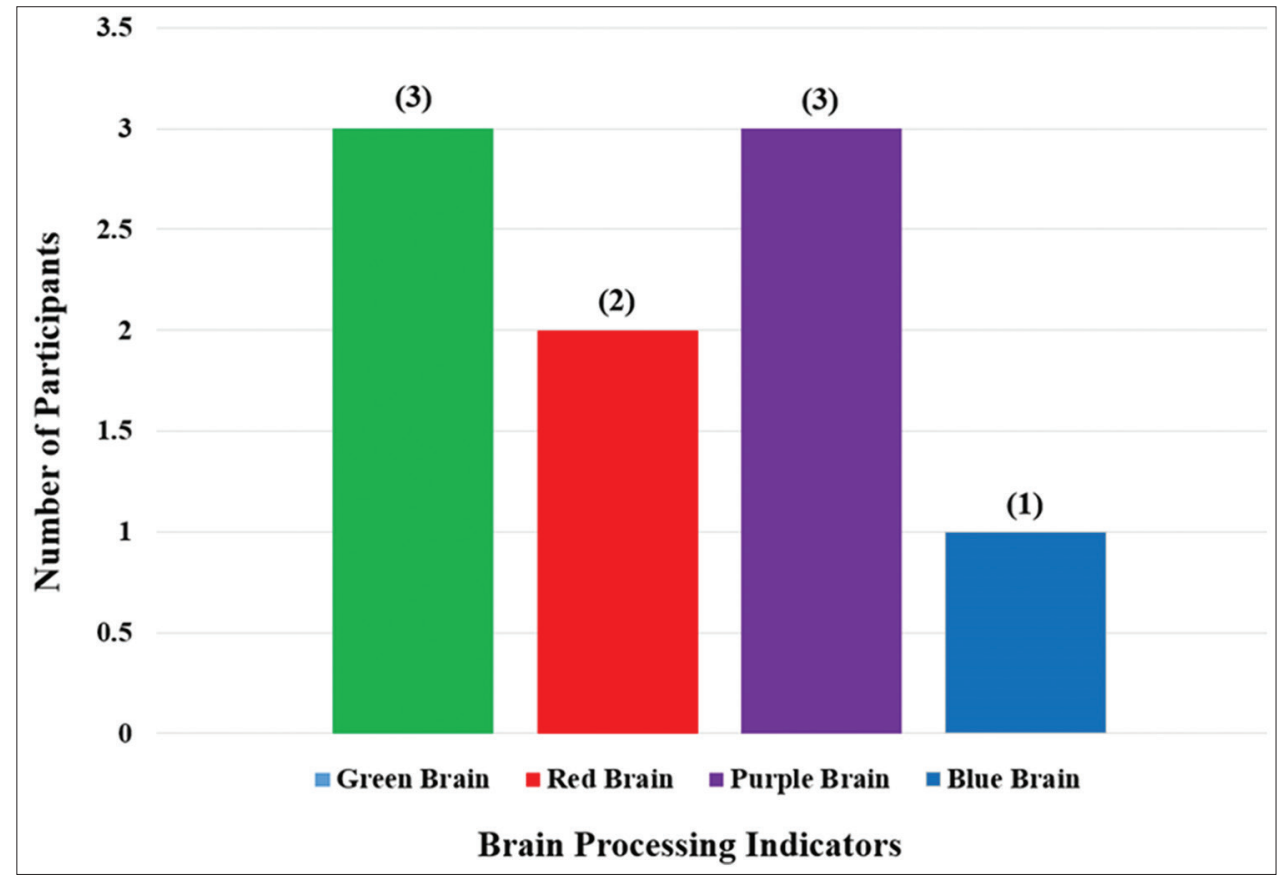

Figure 3: Red Brain processing participants with potentially miscommunication to those participants who were identified as Green, Red, Purple, and Blue Brain processing

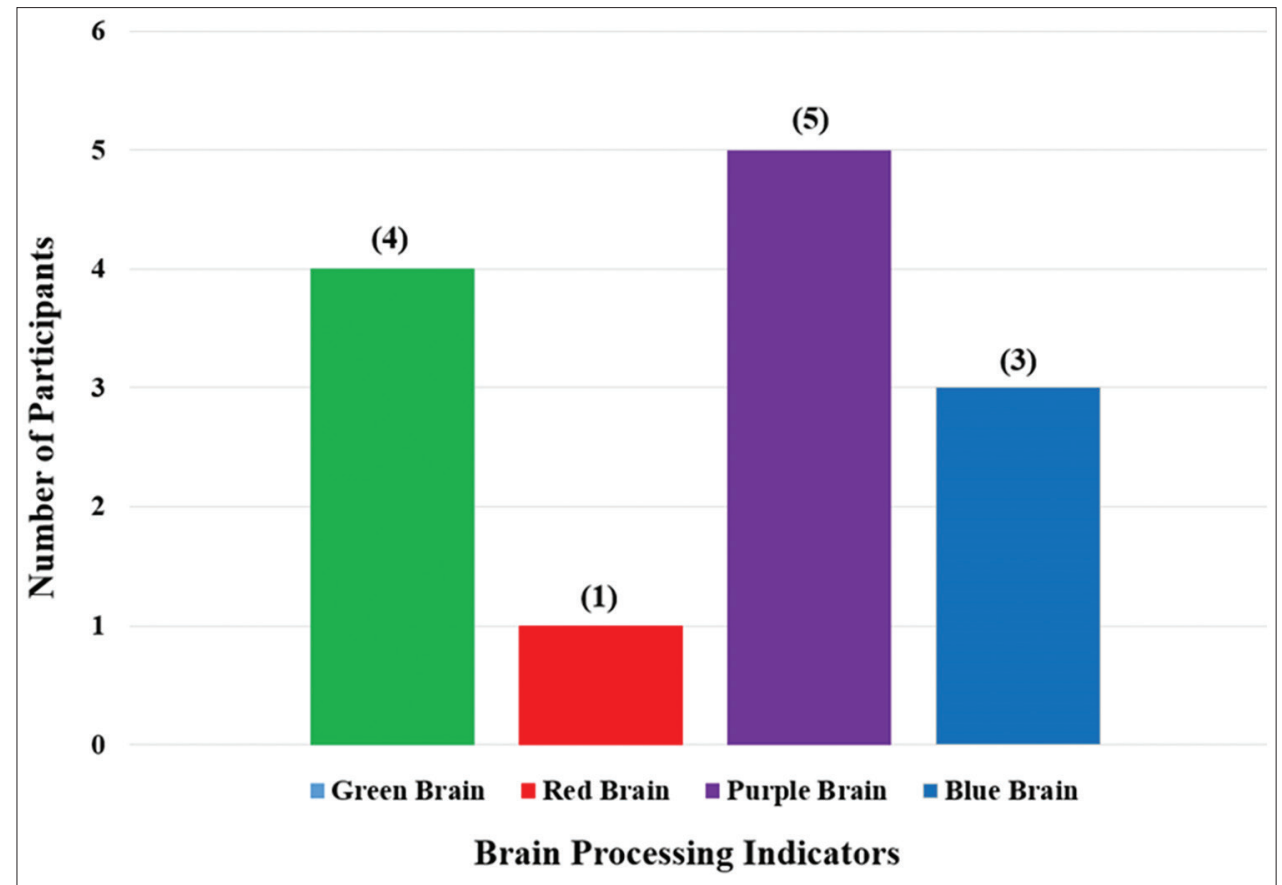

Figure 4: Purple brain processing participants with potentially miscommunication to those participants who were identified as Green, Red, Purple, and Blue Brain processing

\section{Green Brain processing indicator}

Of the twenty-eight participants who were identified as Green Brain processing (Figure 1), these twenty eight Green Brain processing participants might have a high primary miscommunication with Purple Brain processing participants (15 persons), Green Brain processing participants ( 6 persons), Red Brain processing participants
( 3 persons), and Blue Brain processing participants (5 person), respectively (Table 2 and Figure 5).

According to figure 5, the graph shows a highest concentration in Purple section followed by Green sections, while similar concentration in Red and Blue sections, implying that these twenty-eight Green Brain processing participants might have 


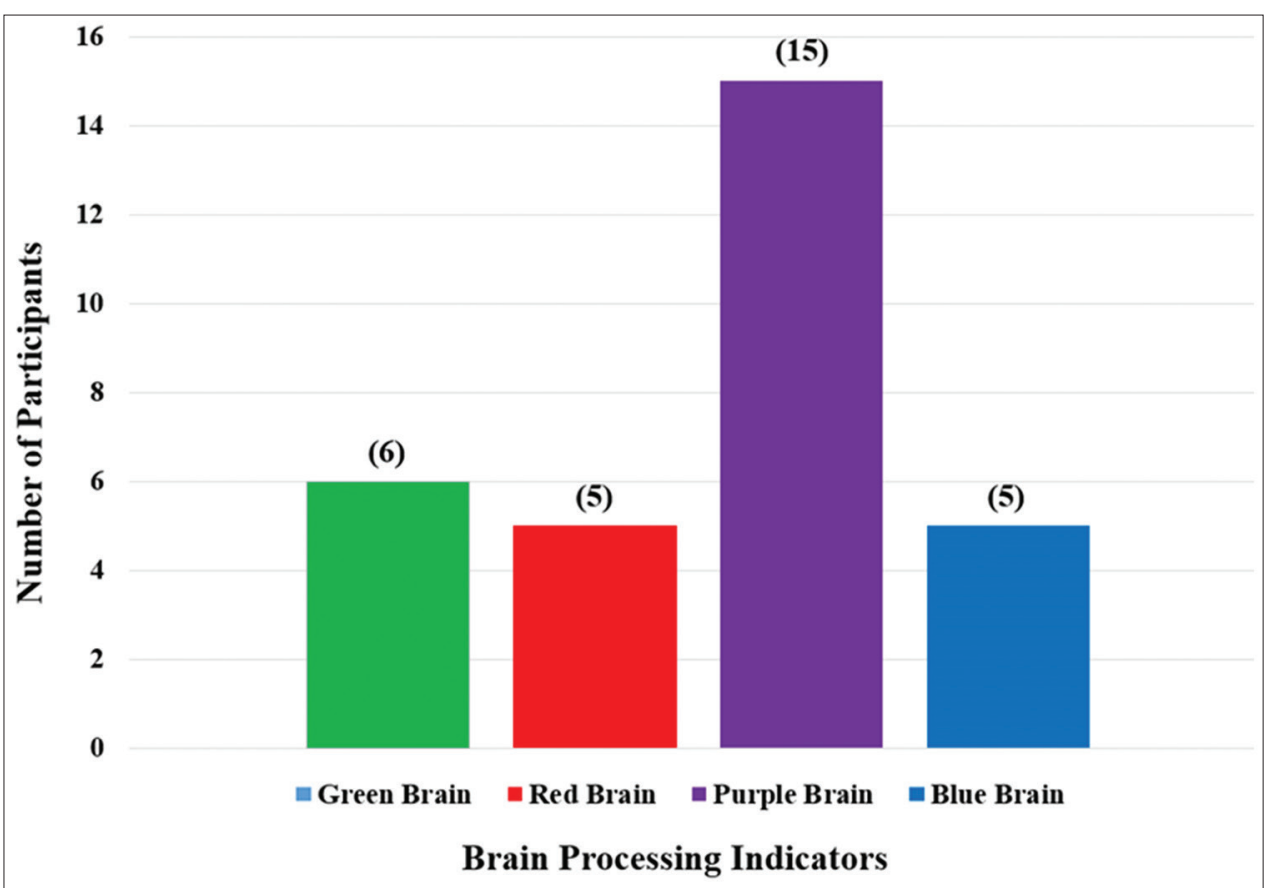

Figure 5: Green brain processing participants with potentially miscommunication to those participants who were identified as Green, Red, Purple, and Blue Brain processing

most difficult time in relating to participants with Purple orientation followed by all Green, Blue, and Red orientations, respectively. In addition, these twenty-eight Green Brain processing participants might be misinterpreting their action process based on their own views instead of leveraging on their strengths to Purple, Green, Red, and Blue Brain processing participants, respectively.

Regarding to the application of colored brain communication to working environment, Carmazzi ${ }^{1}$ symbolized different colors of the brain in classifying the genetic neuroprocessing in such a way that each person experiences their environment and takes in and interprets information resulting in specific ways of perceiving and evaluating events and situations. Carmazzi ${ }^{1}$ identified four key components of so called "Effective Work Environments" including (a) communication and perception gaps creating reactionary behavior that affect personal and group effectiveness, (b) contribution of perceptional variety implying that the way employee add a different perspective to the group to enhance results and problem solving, (c) work processes that that can complement or deter the effectiveness of a work group and how to overcome them, and (d) employee's capacity to adapt to and understand others perceptions and communication processes, respectively ${ }^{1,7,8}$. Carmazzi then classified four Ambiguity relief processes including (a) chaotic processing named as Green Brain, (b) linear processing named as Red Brain, (c) relational processing named as Purple Brain, and (d) intuitive processing named as Blue Brain, respectively, based on genetic and cannot be changed. ${ }^{1,7,8}$ These four colors healthy processes classification simplify them through the so called "Colored Brain" model..$^{7,8}$ Therefore, awareness of these brain processes by participants in the present study could help their team members adopt a cooperation attitude instead of trying to change other team members according to their own processes that might create frustration on both sides. This study concludes the effect of communication and brain processing indicator on the working environment of financial officers in Thailand. The limitations of this study and the directions of future research are still needed for further consideration.

\section{CONCLUSION}

This study explored the relationship between brain processing indicator and communication of financial officers in Thailand. It is the first study to identify the effects of brain processing indicator and communication on the leadership and working environment by using the Colored Brain Communication Inventory in Thailand. Referring to the Colored Brain model, any awareness by participants might help their team members adopt a cooperation attitude instead of trying to change others beyond their own processes which creating frustration on both sides.

\section{ACKNOWLEDGEMENT}

We thank all of the participants who involved in this study as main data provider. The author would like to 
express sincere appreciation to Lily Lau, Colored Brain Communication and Directive Communication Accredited Master Trainer for supporting this research.

\section{REFERENCES}

1. Carmazzi FA. New Research Identifies the Brain's Clarity Getting Process has Foundations in Genetic Neuroscience. EC Neurology 2018; 10(2):20-21.

2. Carmazzi FA. The colored brain communication inventory. 2008 [Online]. Available: www.directive communication.

3. Seagal $\mathrm{S}$ and Horne H. Human Dynamics: A New Framework for Understanding People and Realising the Potential in Our Organizations, Cambridge: Pegasus Communications, 1997.

4. Hoschl C and Kozeny J. Predicting academic performance of medical students: The first three years. The American Journal of Psychiatry. 1997; 154(6): 87-92.
5. Horak E and Du Toit JW. A study of the thinking styles and academic performance of civil engineering students. Journal of the South African Institution of Civil Engineering. 1999; 44(3): 18-24.

6. Carmazzi FA. Colored brain communication inventory. Directive Communication International, 2008.

7. Carmazzi FA. The colored brain communication field manual: Practical applications of directive communication psychology and the colored brain to work, leadership, business and relationship. Directive Communication International, 1999.

8. Carmazzi FA. Colored brain communication card. Directive Communication International, 2004.

9. Chin FCJ, Ooi ML and Yip MW. The Effects of Colored Brain Communication and Brain Processing Interpretation on the Academic Performance of Students: A Literature Review. Int J Inf Educ Technol 2016; 6(12): 945-948.

10. Carmazzi FA. Observation and Genetic Foundations of the Brain's Clarity Achieving "Ambiguity Relief" Processes. Theranostics Brain Disord. 2017; 2(3): 555586.

Authors Contribution:

PS-Concept and design of the study, manuscript preparation, statistically analyzed and interpreted, critical revision of the manuscript; AFC-Concept and design of the study.

Work attributed to:

Brain Science and Engineering Innovation Research Group, School of Anti-Aging and Regenerative Medicine, Mae Fah Luang University, Bangkok 10110, Thailand.

Orcid ID:

Dr. Phakkharawat Sittiprapaporn-10 https://orcid.org/0000-0002-4103-9396

Arthur F. Carmazzi- id htps://orcid.org/0000-0001-5217-449X

Source of Support: Brain Science and Engineering Innovation Research Group, Mae Fah Luang University (MFU-grant), Conflict of Interest: None. 